\title{
Comprehensive Analysis of Proteasomal Complexes in Mouse Brain Regions Detects ENO2 as a Potential Partner of the proteasome in the Striatum
}

Khosrow Rezvani ( $\square$ khosrow.rezvani@usd.edu )

University of South Dakota Sanford School of Medicine https://orcid.org/0000-0003-1963-2211

Niki Esfahanian

McMaster University

Morgan Nelson

University of North Carolina Project

Rebecca Autenried

University of lowa Health Science Library: The University of lowa

Scott Pattison

University of South Dakota Sanford School of Medicine

Eduardo Callegari

University of South Dakota Sanford School of Medicine

\section{Research Article}

Keywords: 26S proteasome, hippocampus, striatum, gamma enolase (ENO2), gradient fractionation

Posted Date: February 17th, 2021

DOl: https://doi.org/10.21203/rs.3.rs-232942/v1

License: (c) (i) This work is licensed under a Creative Commons Attribution 4.0 International License.

Read Full License 


\section{Abstract}

Defects in the activity of the proteasome or its regulators are linked to several pathologies including neurodegenerative diseases. We hypothesize that proteasome heterogeneity and its selective partners vary across brain regions and have a large impact on proteasomal catalytic activities. Using neuronal cell cultures and brain tissues obtained from mice, we compared proteasomal activities from two distinct brain regions affected in neurodegenerative diseases, the striatum and the hippocampus. The results indicated that proteasome activities and their responses to proteasome inhibitors are determined by their subcellular localizations and their brain regions. Using a iodixanol gradient fractionation method, proteasome complexes were isolated, followed by proteomic analysis for proteasomal interaction partners. Proteomic results revealed gamma enolase (ENO2), a known proteasome partner, has more affinity to proteasome complexes purified from the striatum than to those from the hippocampus. These results highlight a potential key role for non-proteasomal partners of proteasome regarding the diverse activities of the proteasome complex recorded in several brain regions.

\section{Full Text}

Due to technical limitations, full-text HTML conversion of this manuscript could not be completed. However, the manuscript can be downloaded and accessed as a PDF.

\section{Figures}

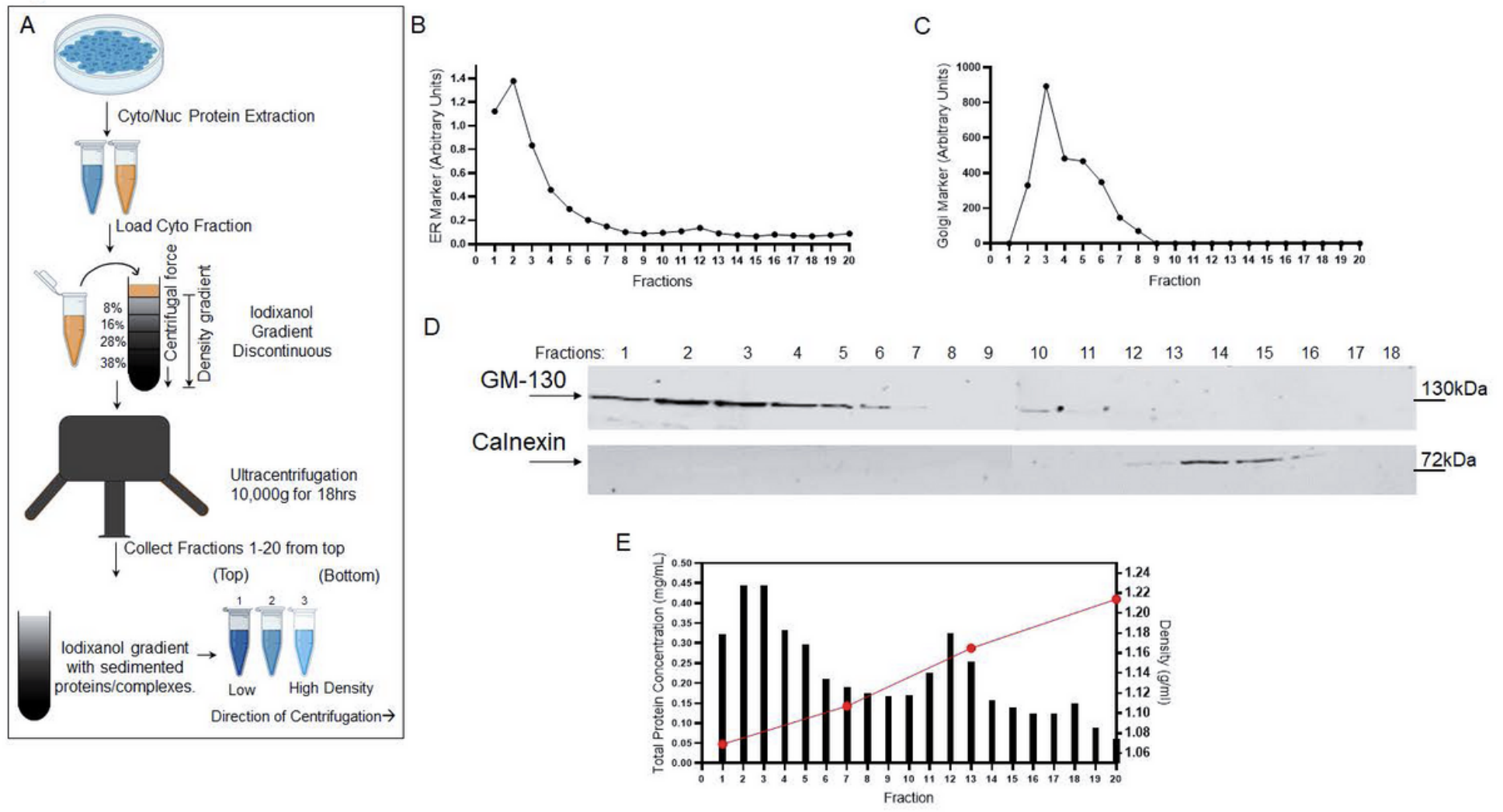


Figure 1

lodixanol gradient fractionation of proteasome complexes and quality control tests. A. Overview of the experimental study. Cytoplasmic and nuclear lysates were fractionated via iodixanol gradient ultracentrifugation and separated into twenty fractions. B and C. Sedimentation of arylesterase (endoplasmic reticulum markers) and leucine aminopeptidases (Golgi apparatus markers) according to their densities verified that iodixanol gradients can provide satisfactory resolution of membrane particles and protein complexes in the cytoplasm segment of mouse striatum cell line. D. GM-130 (Golgi marker) and Calnexin (ER marker) revealed locations of Golgi and ER, respectively. E. Density of fractions $(\mathrm{g} / \mathrm{ml})$ as well as total protein concentration $(\mathrm{mg} / \mathrm{ml})$ of collected fractions were determined in the cytoplasm segments of cells.

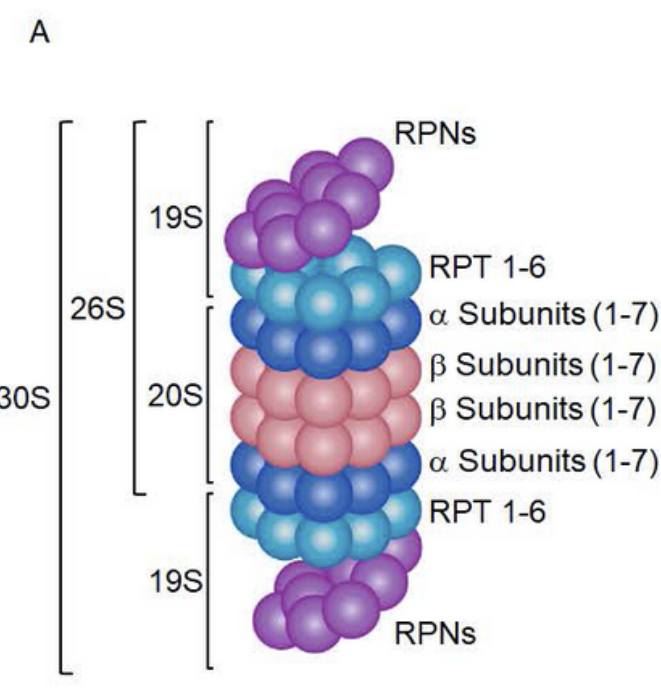

$\mathrm{H}$
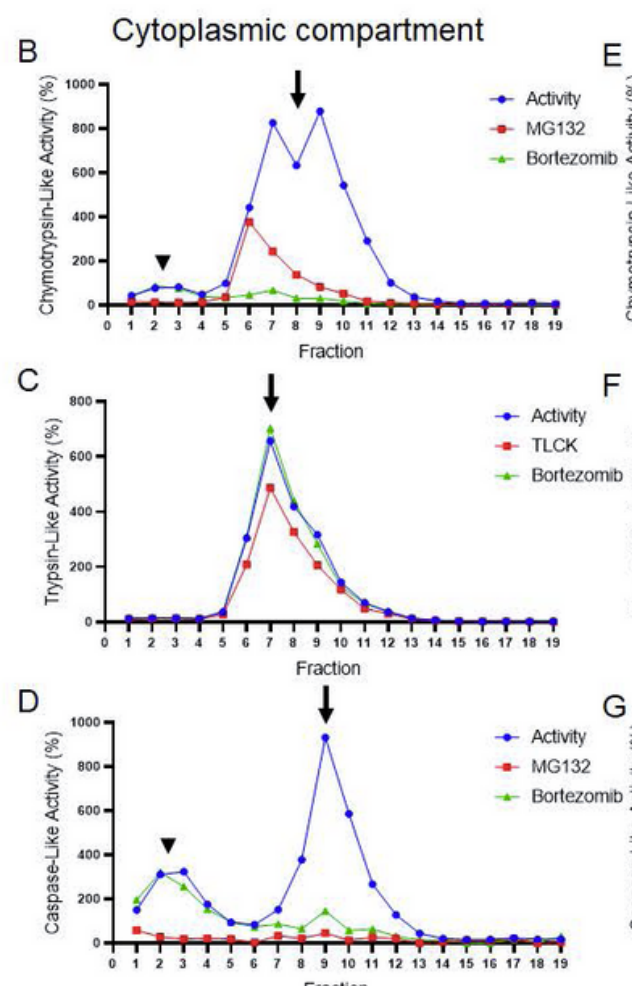

Fraction
Nuclear compartment
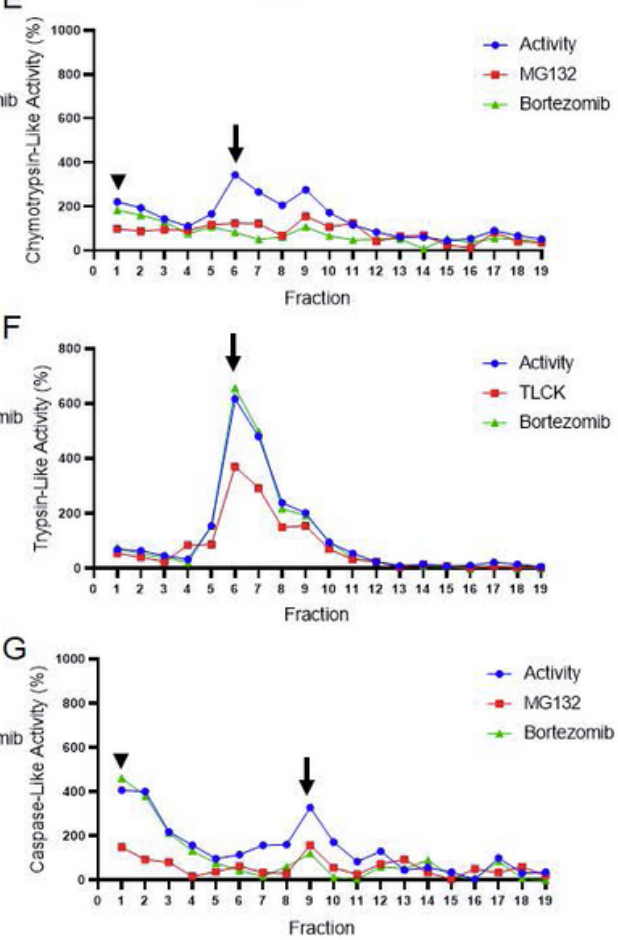

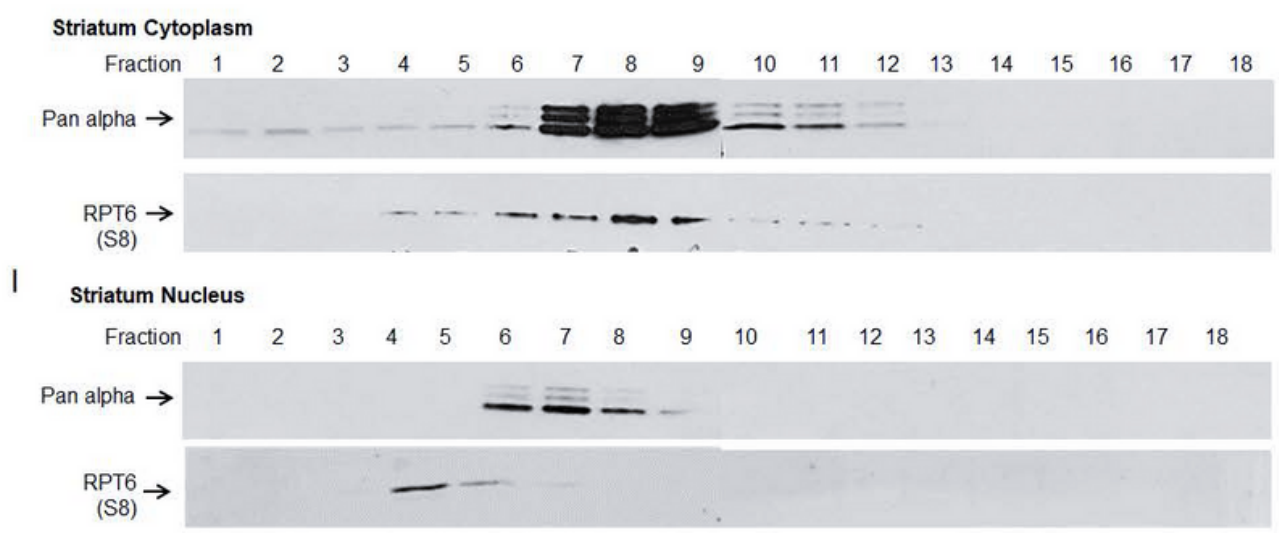

Figure 2

Proteolytic activity and inhibitor response of the proteasome in a mouse striatum-derived cell line. A. Schematic diagram of the 20 S proteasome catalytic complex flanked by the $19 \mathrm{~S}$ regulatory subunit made 
by 6 ATPase subunits (RPT-1-6) and regulatory particles of non-ATPase (RPN) subunits. B-D: Cytoplasmic and E-G: nuclear lysates of mouse striatum-derived cell were subjected to iodixanol gradient ultracentrifugation. Subsequently, samples were analyzed for proteasomal catalytic activities with and without the proteasome inhibitors MG132, TLCK, and bortezomib. Arrowheads represent potential nonspecific proteasomal activities while arrows show peaks of three proteasomal activities recorded in collected fractions. $\mathrm{H}-\mathrm{l}$ : An equal volume of each fraction (Cytoplasm and nucleus) was subjected to SDSPAGE followed by WB using anti-pan alpha (20S proteasome) and anti-RPT6 (19S subunit-S8 ATPase) antibodies to illustrate the distribution of proteasome complexes in the collected fractions.
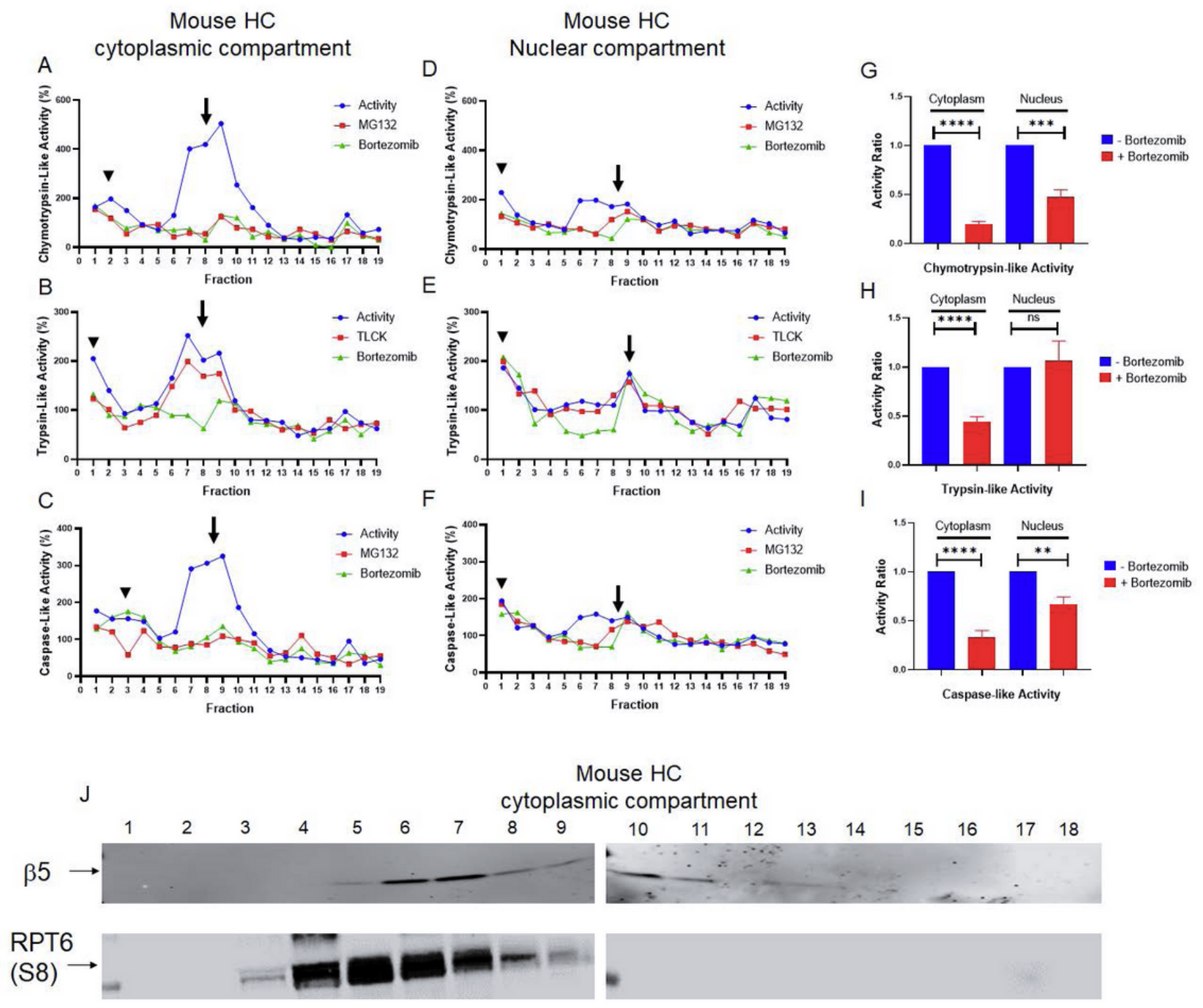

Mouse HC

\section{Figure 3}

Proteasome catalytic activities in mouse striatum. A-C: Cytoplasmic and D-F: nuclear lysates from mouse striatum tissue were subjected to iodixanol gradient ultracentrifugation. Collected fractions were treated with MG132, bortezomib, and TLCK, and activities of the proteasome complex were measured.

Proteasome activities indicate differences between the cytoplasm and the nucleus. Arrowheads represent non-specific proteasome activities and arrows show peaks for three proteasomal activities. G-I: Based on 
the distribution pattern of proteasome activity in the collected fractions, proteasomal peaks were individually compared for the chymotrypsin-, trypsin-, and caspase-like activities in both cytoplasm and nucleus compartments with and without bortezomib treatments. Graphs represent the mean $\pm S D(P \star \star \star *$ $<0.0001, P^{* * *}<0.001, P^{* *}<0.01$ and $\left.P^{*}<0.05\right)$. This set of experiments was conducted on three male mice while corresponding assays were performed in triplicate. Panel $\mathrm{J}$ : Cytoplasmic fractions were subjected to WB using $₫ 5$ (PSMB5) and RPT6 antibodies
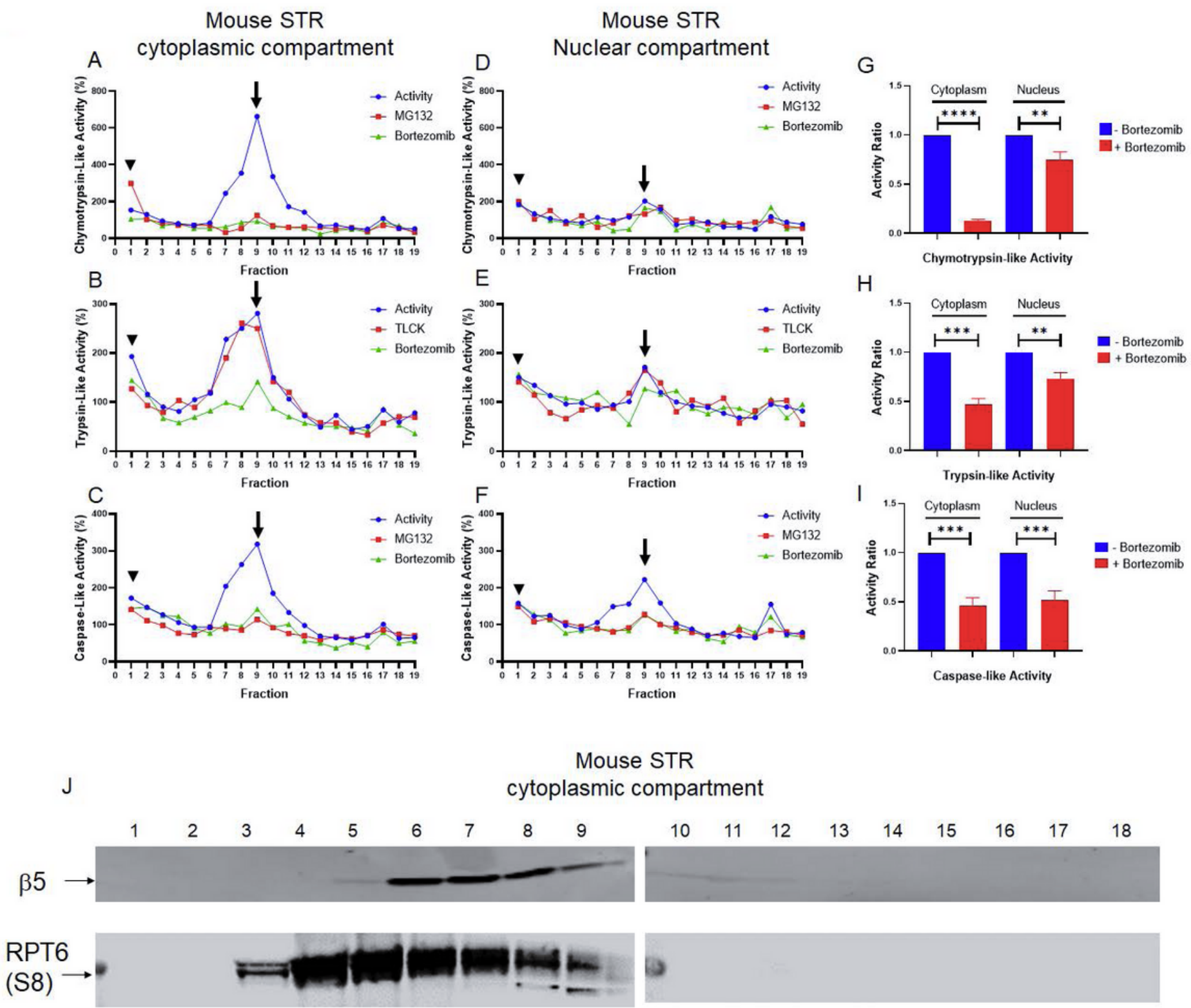

\section{Figure 4}

Proteasome catalytic activity in mouse hippocampus. A-C: Cytoplasmic and D-F: nuclear lysates from mouse hippocampus tissue were subjected to iodixanol gradient ultra-centrifugation. Collected fractions were treated with MG132, bortezomib, and TLCK, and activities of the proteasome complex were measured. Proteasome activities indicate differences between the cytoplasm and the nucleus. Arrowheads represent non-specific proteasome activities and arrows show peaks for three proteasomal activities. G-I: Based on the distribution pattern of proteasome activity in the collected fractions, 
proteasomal peaks were compared for the chymotrypsin-, trypsin-, and caspase-like activities in both cytoplasm and nucleus compartments with and without bortezomib treatments. This set of experiments was conducted on three male mice followed by proteasomal assays in triplicate. Graphs represent the mean \pm SD calculated $\left(P^{* * * *}<0.0001, P^{* * *}<0.001, P^{* *}<0.01\right.$ and $\left.P^{*}<0.05\right)$.. Panel J: Cytoplasmic fractions were subjected to WB using $₫ 5$ (PSMB5) and RPT6 antibodies.
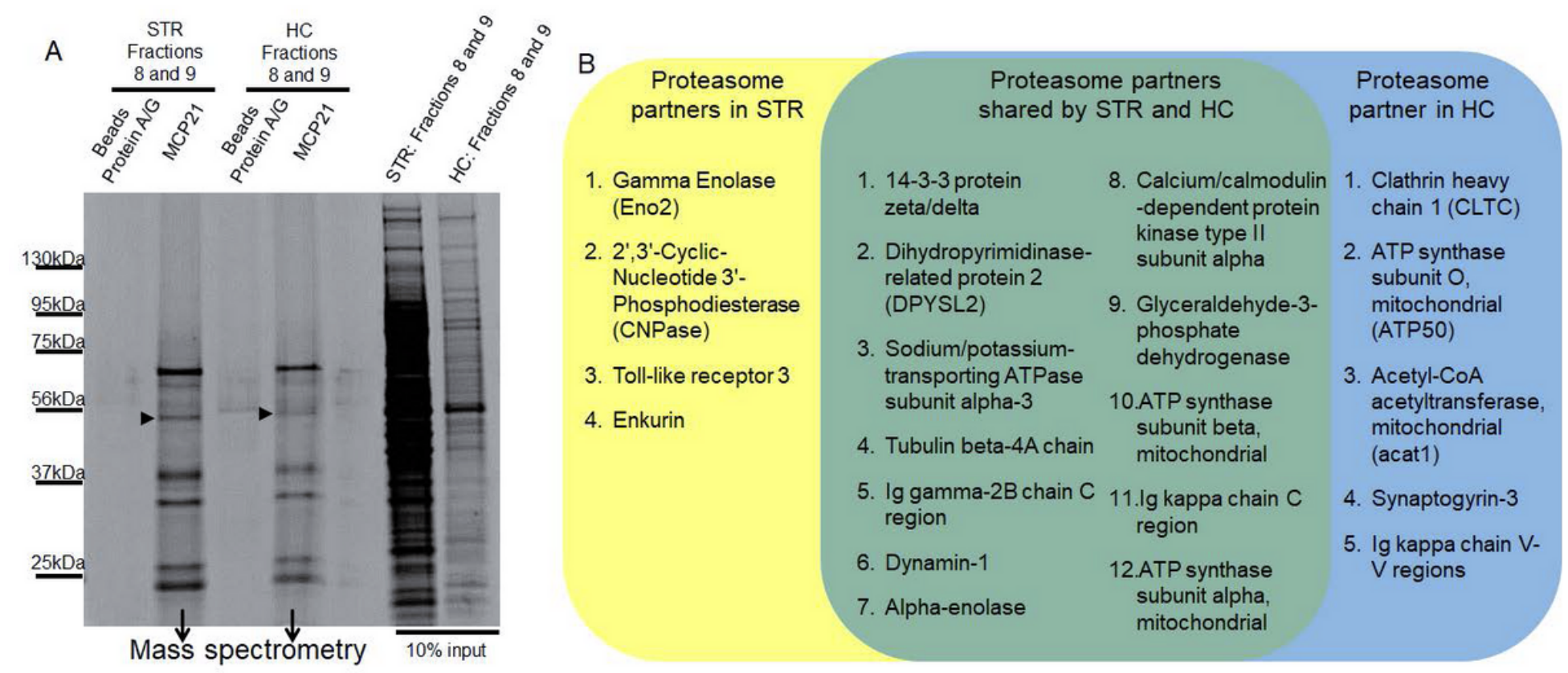

\section{Figure 5}

Proteasome associated partners in mouse striatum and hippocampus found by an affinity purification method and a mass spectrometry approach. A. Fractions 8 and 9 enriched with proteasome complexes after iodixanol gradient fractionation of mouse striatum (STR) and hippocampus (HC) were subjected to IP using MCP21 antibodies immobilized on agarose beads. Control groups were protein A/G beads with mouse IgG serum. Associated proteins pulled down by proteasome complex (dominantly $20 \mathrm{~S}$ core) were separated by a moderate salt buffer and subjected to SDS-PAGE followed by mass spectrometry (details in Materials and Methods section). B. Mass spectrometry results identified known and unknown proteins associated with proteasome complexes enriched from mouse hippocampus and striatum tissues. A group of proteins were found to be associated with only one specific tissue, including Eno2, which was only detected in the STR brain region. The arrowheads in Fig.5A point to a band below $56 \mathrm{kDa}$ which likely is Eno2 protein pulled down dominantly in striatum fractions. 


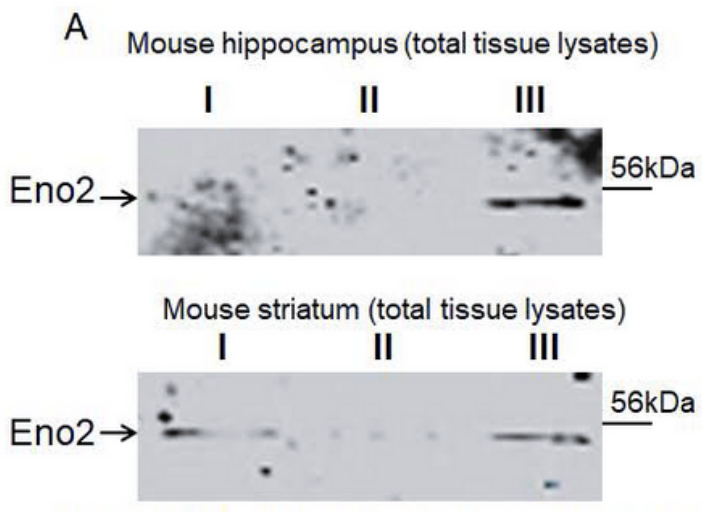

Lane I: MCP21 (alpha2) immobilized on agarose beads.

Lane II: IgG immobilized on agarose beads

Lane III: Inputs (10\%)

C

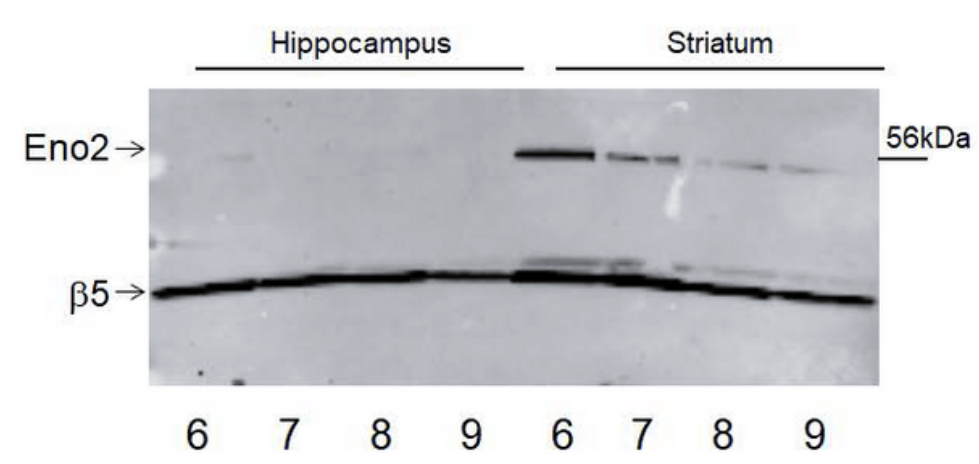

Fractions with enriched proteasomal activities
Mouse hippocampus or striatum (total tissue lysates)

B $\quad \frac{\mathrm{HC}}{\mathrm{I} \text { II }} \stackrel{\text { STR }}{\text { III IV }} \frac{\text { Input }}{\mathrm{V} \mathrm{VI}}$

Eno2 $\rightarrow$

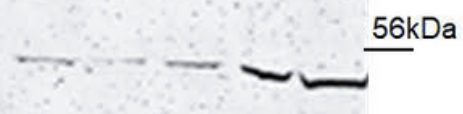

RPT6

Heavy

chain IP:RPT6, IB: Eno-2

I: IgG immobilized on magnetic beads.

II: Anti-Rpt6 antibody immobilized on magnetic beads.

III: IgG immobilized on magnetic beads.

IV: Anti-Rpt6 antibody immobilized on magnetic beads.

$\mathrm{V}$ : Striatum

VI: Hippocampus (HC).

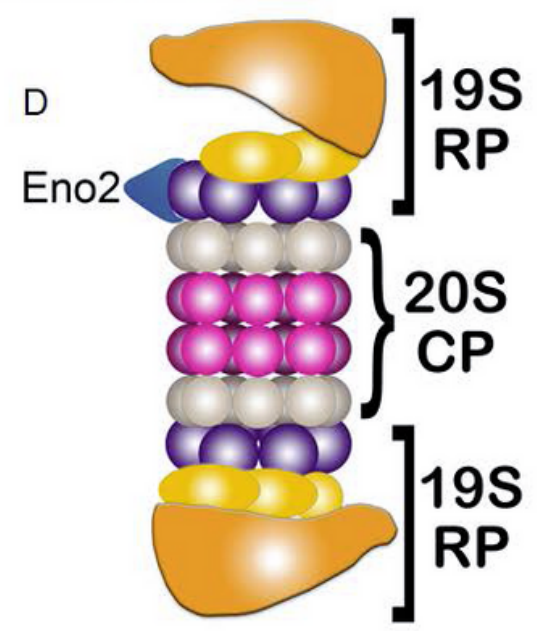

\section{Figure 6}

Enolase 2 (Eno2-gamma enolase) protein has a high binding affinity to mouse striatum proteasome. A. Hippocampus and striatum tissue lysates were subjected to immunoprecipitation using MCP21 (antibody against the $20 \mathrm{~S}$ proteasome). Lane 1 demonstrates pulled-down $20 \mathrm{~S}$ in association with the striatum Eno2 (bottom gel), while no association is observed with the hippocampus Eno2 (top gel). The last lanes (inputs) in the top and bottom gels in panel A confirmed the presence of Eno2 proteins in both mouse STR and HC. B: In another set of experiments, both mouse HC and mouse STR tissues were subjected to IP using RPT6 antibodies immobilized on agarose beads. Control groups were beads immobilized with mouse IgG. After IP, pulled-down proteins were subjected to WB. Interestingly, Eno2 showed association with both HC and STR 26S proteasomes pulled down by anti-RPT6 antibodies. However, measurement of pixels in lanes II and IV with UN-SCAN-IT gel (version 6.1) showed a pixel total of 9432 in lane II (Hippocampus) versus a pixel total of 12401 in lane IV (striatum). These findings indicate that Eno2 shows more affinity to mouse striatum $26 \mathrm{~S}$ proteasome versus mouse hippocampus proteasome (30\% higher affinity). C. Finally, fractions 6 to 9 (high peaks for proteasome complexes collected from 
hippocampus and striatum tissues were subjected to WB using Eno-2 and $₫ 5$ antibodies. Eno2 substantially cofractionated with striatum proteasome. D. These results suggest high affinity of Eno2 to striatum proteasome complexes.

\section{Supplementary Files}

This is a list of supplementary files associated with this preprint. Click to download.

- Figs1.png

- Figs2.png 